\title{
Nomogram for predicting the risk of bone metastasis in breast cancer: a SEER population-based study
}

\author{
Li-Jun Ye ${ }^{1,2 \#}$, Huan-Dan Suo ${ }^{1 \#}$, Chun-Yan Liang ${ }^{3}$, Lei Zhang ${ }^{1}$, Zi-Ning Jin ${ }^{1}$, Cheng-Ze Yu ${ }^{4}, B_{0}$ Chen $^{1 \wedge}$ \\ ${ }^{1}$ Department of Breast Surgery, The First Affiliated Hospital of China Medical University, Shenyang, China; ${ }^{2}$ Department of Breast Surgery, \\ Tungwah Hospital of Sun Yat-sen University, Dongguan, China; ${ }^{3}$ Department of Medical Oncology, the Fourth Affiliated Hospital of China, \\ Shenyang, China; ${ }^{4}$ Department of Breast Surgery, Dongguan Kanghua Hospital, Dongguan, China \\ Contributions: (I) Conception and design: LJ Ye, HD Suo, CZ Yu, B Chen; (II) Administrative support: CZ Yu, B Chen; (III) Provision of study \\ materials or patients: LJ Ye, HD Suo, CZ Yu, B Chen; (IV) Collection and assembly of data: LJ Ye, HD Suo, CY Liang, L Zhang, ZN Jin; (V) Data \\ analysis and interpretation: All authors; (VI) Manuscript writing: All authors; (VII) Final approval of manuscript: All authors. \\ \#These authors contributed equally to this work. \\ Correspondence to: Cheng-Ze Yu. Department of Breast Surgery, Dongguan Kanghua Hospital, Dongguan, China. Email: Yuchengze@Sina.com; \\ Bo Chen. Department of Breast Surgery, The First Affiliated Hospital of China Medical University, Shenyang, China. Email: bochen@cmu.edu.cn.
}

Background: Bone is the most common metastasis site of breast cancer. The prognosis of bone metastasis is better than other distant metastases, but patients with skeletal related events (SREs) have a poor quality of life, high healthcare costs and low survival rates. This study aimed to establish an effective nomogram for predicting risk of bone metastasis of breast cancer.

Methods: The nomogram was built on 4,895 adult/female/primary invasive breast cancer patients with complete clinicopathologic information, captured by the Surveillance, Epidemiology, and End Results (SEER) database from 2010 to 2015. Five biological factors (age, grade, histologic type, surgery of breast lesions and subtypes) were assessed with logistic regression to predict the risk of bone metastases. The predictive accuracy and discriminative ability of the nomogram were determined by the Receiver Operating Characteristic (ROC) curves and the calibration plot. Results were validated on a separate 2,093 cohort using bootstrap resampling from 2010 to 2015 as an internal group and a retrospective study on 120 patients in the First Affiliated Hospital of China Medical University from 2010 to 2014 at the same situation as an external group.

Results: On multivariate logistic regression of the primary cohort, independent factors for bone metastases were age, grade, histologic type, surgery of breast lesions and subtypes, which were all selected into the nomogram. The calibration plot for probability of incidence showed good agreement between prediction by nomogram and two observations. The ROC curves presented a good statistical model for risk of bone metastasis, and the corresponding AUC value of the development group, internal validation group and external validation group were $0.678,0.689$ and 0.704 respectively.

Conclusions: The proposed nomogram resulted in more-accurate prognostic prediction for breast cancer patients with bone metastases.

Keywords: Breast cancer; bone metastases; biological factors; nomogram

Submitted Jun 21, 2020. Accepted for publication Oct 21, 2020.

doi: $10.21037 /$ tcr-20-2379

View this article at: http://dx.doi.org/10.21037/tcr-20-2379

\footnotetext{
^ ORCID of Li-Jun Ye: 0000-0001-8703-2053; ORCID of Huan-Dan Suo: 0000-0003-0384-1203; ORCID of Bo Chen: 0000-0002-22162794.
} 


\section{Introduction}

Breast cancer has the highest incidence of female cancer in the world (1). Bone is the most common metastatic site associated with breast cancer, which accounts for $65-75 \%$ of distant metastases among breast cancer patients (2). Bone metastases may occur independently or with visceral metastases, with best survival reported in patients with bone-only metastases $(3,4)$. Median survival for bone-only metastases has been reported to be as high as 72 months in one study (4), but can be substantially less (4-6), and only $20-30 \%$ of patients with breast cancer are expected to achieve 5 year survival post diagnosis of bone metastases $(2,7)$.

The burden of bone metastases for patients is considerable, those patients are prone to develop SREs in the first year that could seriously lower the quality of life, increase healthcare costs, decrease survival rates, and even lead to death $(2,8)$. In recent years, several clinical trials demonstrated that, bone targeting agents (i.e., bisphosphonates, denosumab) were the most effective for SREs prevention in solid tumors, while significantly delaying the time to first SRE and improving patients' quality of life (9). However, the current treatment for bone metastases is palliative (9). Our study aimed to select the patients with high propensity for breast cancer bone metastasis through existing methods, in order to take appropriate intervention measures in time to prevent or delay the occurrence of SREs and prolong their survival time.

We found that there have been few studies involving the correlation between breast cancer metastases with biological factors at present. Moreover, the reported results were contradictory (10-12), remaining some uncertain clinical issues. Press and Wei's studies suggested that luminal A breast cancer was more prone to bone metastasis $(10,11)$, while Otto thought luminal B breast cancer had the highest risk of incidence of bone metastasis (12). Further research will be needed to identify effective factors for predicting risk of bone metastases. Currently, nomograms have been developed in most cancer types (13-15). It has been proposed as an alternative or even as a new standard for diagnosis and/or prediction (16-18). Therefor we attempt to establish a nomogram for breast cancer patients with bone metastases based on clinicopathologic data from SEER database and 120 patients in the First Affiliated Hospital of China Medical University, to determine whether this model provides more-accurate prediction of risk of bone metastases.
Based on the comprehensive treatment and good prognosis, the population of patients diagnosed with stage IV breast cancer is relatively low. It is difficult to collect data of stage IV breast cancer patients, which limits further research. The SEER database, which represents $30 \%$ of the information about cancer patients in the United States (19), is more reliable to show the real situation of most patients. The aim of the nomogram is to predict high-risk of bone metastases and take an effective intervention during followup, so that increase the survival rate and even benefit a few patients clinical cure. We present the following article in accordance with the TRIPOD reporting checklist (available at http://dx.doi.org/10.21037/tcr-20-2379).

\section{Methods}

\section{Patients and study design}

We obtained population-based data from the SEER 18 registry research database (SEER*Stat Version 8.3.6). With the SEER database, we identified 446,807 cases of breast cancer, of which 6,988 eligible patients with distant metastases from January 2010 to December 2015. The time frame was selected because information on specific distant metastatic sites and detailed molecular subtypes from SEER were available only for those who were diagnosed after 2010. Inclusion criteria included: (I) stage IV breast cancer with distant metastases; (II) intact clinicopathologic characteristics; (III) primary invasive breast cancer; (IV) intact metastatic sites; (V) adults (>18 years old); (VI) singlesource breast cancer; (VII) active follow-up; (VIII) female.

The study was conducted in accordance with the Declaration of Helsinki (as revised in 2013). This article does not contain any studies with human participants or animals performed by any of the authors. All procedures performed in study involving human data were extracted freely from the SEER Research Data available to the public online (https://seer.cancer.gov/data/access.html).

The primary cohort of 4,895 breast cancer patients were in the inclusion criteria selected from the SEER database between 2010 and 2015. Those patients were eligible for univariate and multivariate logistic analysis. Internal validation of the developing group was performed using the bootstrap validation approach on 1,000 cases. The internal validation group consisted of 2,093 patients. From 2020 to 2014, an external validation group of 120 patients diagnosed with distant metastases of breast cancer in the First Affiliated Hospital of China Medical University was 
retrospectively studied, using the same inclusion criteria.

\section{Biological factors}

The information including age, surgery of primary site, histologic grade, histologic types, tumor (T), lymph nodes $(\mathrm{N})$, estrogen receptor (ER), progesterone receptor (PR), human receptor epidermal growth receptor-2 (HER-2) and metastatic sites were obtained from the SEER database between 2010 to 2015. The current American Joint Committee on Cancer (AJCC) staging systems: TNM system (versions 6.0 and 7.0) are prognostic systems that stratify cancer patients into different risk groups (20). T0 patients are defined as not having evidence of a primary tumor, according to the TNM system, in both AJCC versions 6 and 7. Patients were stratified as four subtypes: HR+/HER-2-; HR+/HER-2+; HR-/HER-2+ and HR-/ HER-2-. HR+ represents ER+ and/or PR+, while HRrepresents ER- and PR-. According to AJCC guidelines for breast cancer and the Scarff-Bloom-Richardson system, histologic grade was divided into three groups: grade I (3-5 points); grade II (6-7 points) and grade III (8-9 points). The absolute numbers were calculated for breast cancer patients with bone metastases identified at the time of diagnosis. Absolute numbers were also calculated after stratification by age, histologic type, histologic grade, surgery of the primary site, and other factors.

\section{Statistical analysis}

The statistical analyses to identify risk factor were performed using SPSS (version 21.0 for Mac; SPSS, Inc., Chicago, IL, USA). Table 1 presented the clinicopathologic characteristics of developing cohort and validation cohort. Univariate logistic regression was used to determine the biological factors associated with the incidence of bone metastases and those statistically significant variables were included in the multivariate logistic regression analysis. $\mathrm{P}$ value $<0.05$ was considered statistically significant.

A nomogram was formulated based on the results of multivariate analysis using forward stepwise method to select the independent variables and by using the package of 'rms' in R (version 3.5.2, USA). The performance of the nomogram was evaluated by the area under the curve (AUC) value of ROC curve. With the AUC value closer to 1 , the risk prediction became more accurate. The calibration plot of prediction model was used to assess the agreement between observed outcome frequencies and predicted probabilities. The calibration plot was designed to descript the relationship between the predicted probability of bone metastasis and the observed frequencies of bone metastasis in the validation set by fitting a polynomial logistic function. And it also calculates the $95 \%$ confidence interval (95\% CI) through 1,000 repeated bootstrapping. When the $95 \%$ CI does not cross the bisector, statistically significant deviation from the bisector vector occurs. Wider confidence intervals are considered as a higher degree of uncertainty, for tiny proportion of patients is at the specific risk interval. Small $\mathrm{P}$ value $(\mathrm{P}<0.05)$ provides evidence that the prediction model's calibration is not perfect. Large $\mathrm{P}$ value of calibration test suggests that there is not strong evidence of model's lack of fit.

\section{Results}

We selected 446,807 breast cancer patients from SEER database (19), and the breast cancer metastatic rate was $4.912 \%$, which was in agreement to the $6 \%$ reported by Hagberg (21). Moreover, breast cancer with bone metastases accounted for $63.9 \%$ of metastatic breast cancer cases, which is consistent with $65-75 \%$ incidence of bone metastases among breast cancer patients worldwide (2). The overall median follow-up time was 22 and 47.4 months for SEER data and clinical data. Breast cancer patients with bone-only metastases had a better prognosis than other metastatic breast cancer patients. There were substantive differences in overall survival (OS) $\left(\chi^{2}=274.0, \mathrm{P}<0.001\right)$ among the three metastatic groups of SEER database. The median OS time was 43, 22 and 31 months respectively in bone-only, bone + visceral and extraskeletal metastatic groups.

The study involved 7,108 cases of breast cancer $(4,895$ cases in the SEER development group, 2,093 cases in the SEER validation group and 120 cases in the clinical validation group). The demographic and clinicopathologic characteristics of patients in the primary and validation cohorts are listed in Table 1 .

\section{Nomogram development}

The results of the univariate and multivariate logistic analysis were listed in Table 2. Upon univariate and multivariate analysis of the developing group, the independent prognostic factors for bone metastasis were age, surgery of the primary site, histologic grades, histologic types and breast cancer subtypes $(\mathrm{P}<0.001)$, while the $\mathrm{T}$ and $\mathrm{N}$ were not correlated 
Table 1 Demographic and clinicopathologic characteristics of the development group and the validation groups

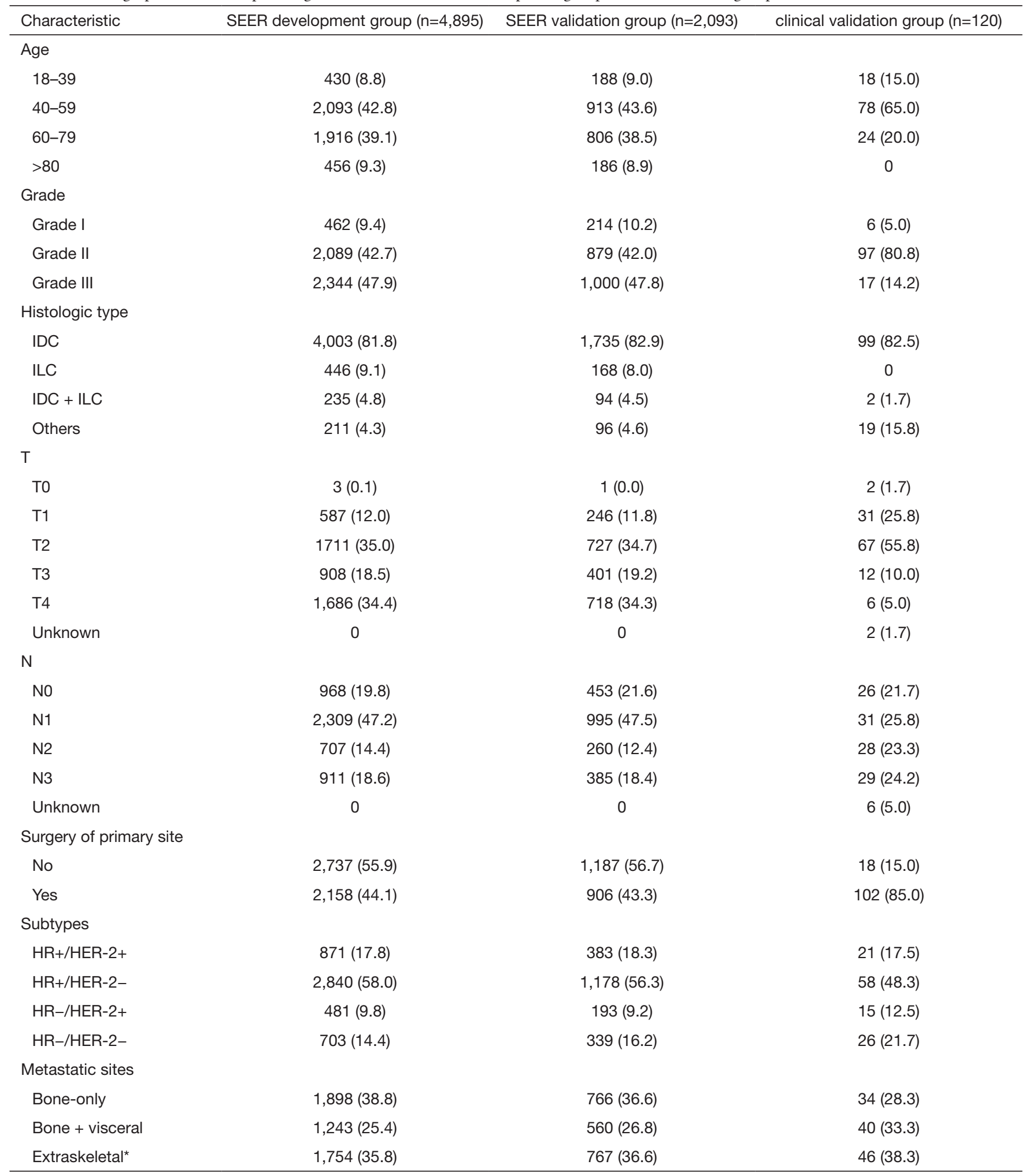

*, extraskeletal metastatic sites indicate metastases of lung, brain, liver or distant lymph nodes. IDC, infiltrative ductal carcinoma; ILC, infiltrative lobular carcinoma; T, tumor; N, lymph nodes; HR, hormone receptor; HER-2, human epidermal growth receptor-2; - denotes negative, + denotes positive. 
Table 2 Univariate and multivariate logistic regression models in the development group

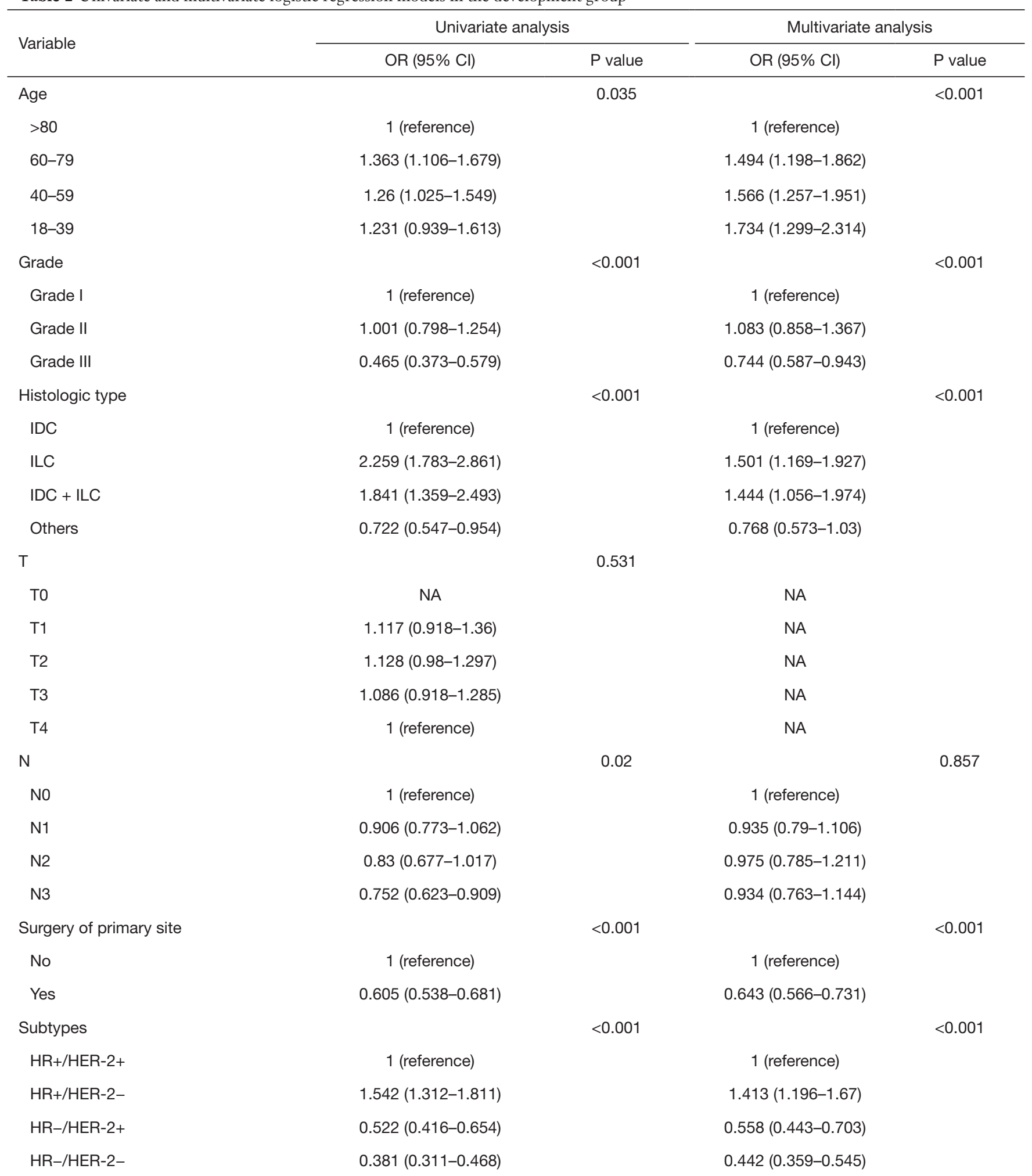

IDC, infiltrative ductal carcinoma; ILC, infiltrative lobular carcinoma; T, tumor, N, lymph nodes; HR, hormone receptor; HER-2, human epidermal growth receptor-2; - denotes negative; + denotes positive. 


Points
Age
Surg__Prim_Site
Subtypes
Histologic_grade
Histologic_type
Total Points
Linear Predictor
Diagnostic possibility

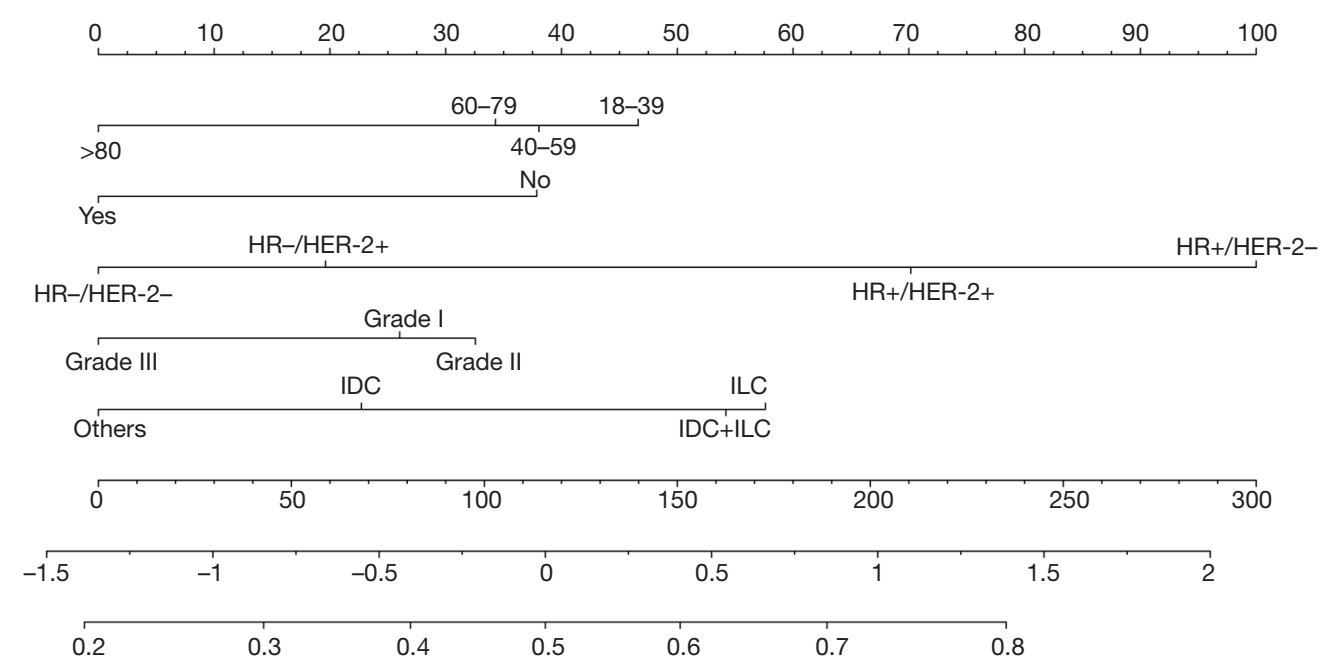

Figure 1 Nomogram to predict the probability of bone metastasis in the patient with breast cancer.

statistically significant to bone metastases.

\section{Risk predictive nomogram}

The individualized nomogram prediction model that integrated all significant independent factors for bone metastases in the developing group is shown in Figure 1. Based on the nomogram, we can get the score corresponding to each prediction indicator, and the total score is corresponding to the probability of risk prediction of bone metastases.

As shown in Figure 1, the highest probability of subtype is $\mathrm{HR}+/ \mathrm{HER}-2-(100$ points), it Intuitively shows that HR+/HER-2- breast cancer is more prone to bone metastasis than triple-negative breast cancer, followed by the HR+/HER-2+ subtype (61 points). The susceptible factors associated with increasing incidence of bone metastases: young patients, HR+ subtype, low histological grade, infiltrative lobular carcinoma (ILC), and primary breast site without surgery.

\section{Nomogram validation}

The validation of the model was based on discrimination and calibration. We drew the ROC curves of predicted probability and calculated the AUC values in the development and validation group. The AUC values for the nomogram established in the development group internal validation group and external validation group were $0.678,0.689$ and 0.704 (Figure $2 A, B, C$ ), suggesting that the nomogram was a useful predictive model.

The $95 \%$ CI of calibration belt in both development and validation groups did not cross the diagonal bisector line, and the $\mathrm{P}$ value were $0.982,0.662$ and 0.245 respectively (Figure $3 A, B, C$ ). Therefore, the calibration plot showed good agreement between prediction and observation in the probability of bone metastases.

\section{Discussion}

Bone metastasis is not life-threatening, but the SREs can lead to a poor prognosis for breast cancer patients and even a decrease of their survival time (5). We developed a helpful nomogram based on common clinicopathologic factors available from pathology reports. We aim to filter out highrisk patients and take effective treatment timely, which will make it possible for clinical benefit. Although some models have been recently developed to risk prediction, the population studied in academic cancer centers with breast cancer is relatively small compared with SEER database, which limits the veracity of the model (22). Ouldamer has verified that the MDACC model failed to confirm its generalizability to predict the risk of bone metastasis in an independent stage I-III breast cancer population (22). External and internal statistics validated the nomogram (AUC value was 0.689 and 0.704 ), attesting to the strength of our model built on the large number of patients.

A multivariate logistic regression analysis of the nomogram revealed that $\mathrm{HR}+$ subtypes was a significant predictor for a high-risk bone metastasis. Yann Delpech published a model without molecular subtypes classification, but our prediction model made it more comprehensive (23). We identified that HR+/HER-2 - subtypes and HR+/ HER2 + subtypes were more susceptible to bone metastasis, 

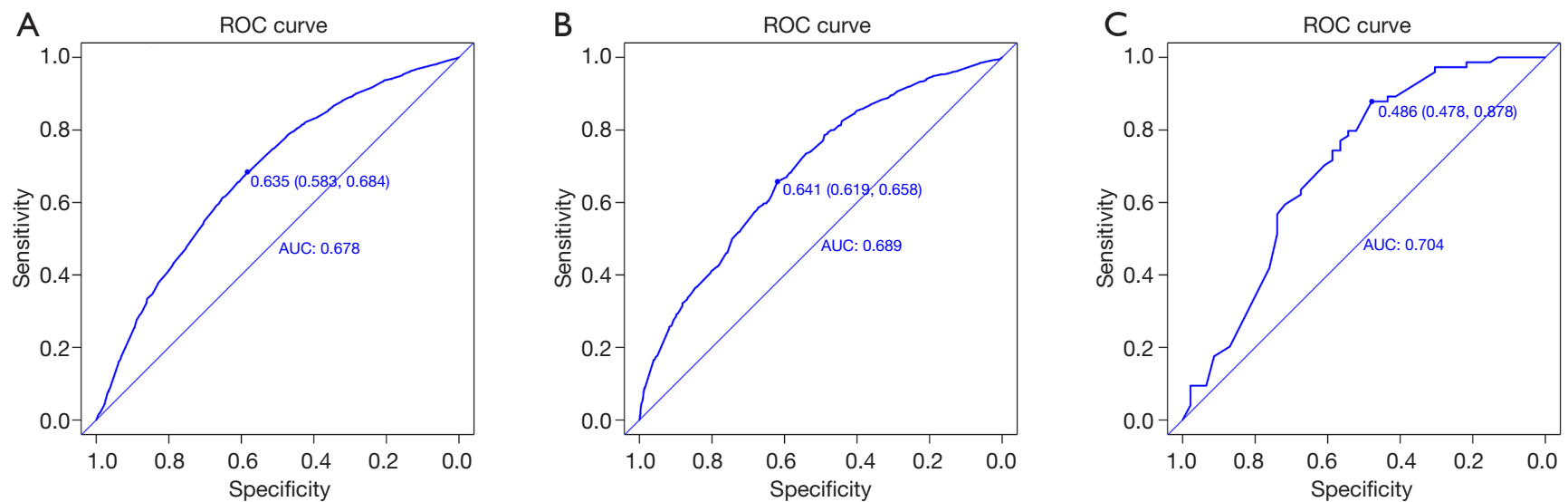

Figure 2 ROC curves for validating the discrimination power of the nomogram. (A) SEER development group; (B) Seer validation group; (C) clinical validation group. $\mathrm{AUC}=0.678$ vs. 0.689 vs. 0.704 .

A

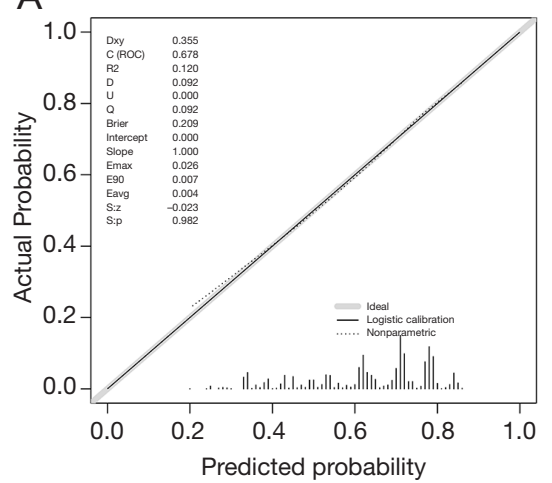

B

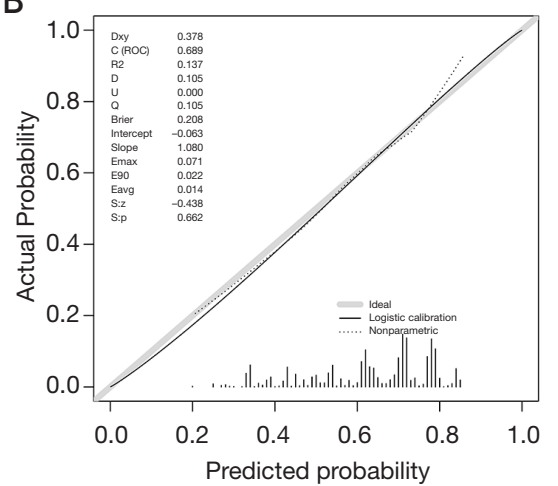

C

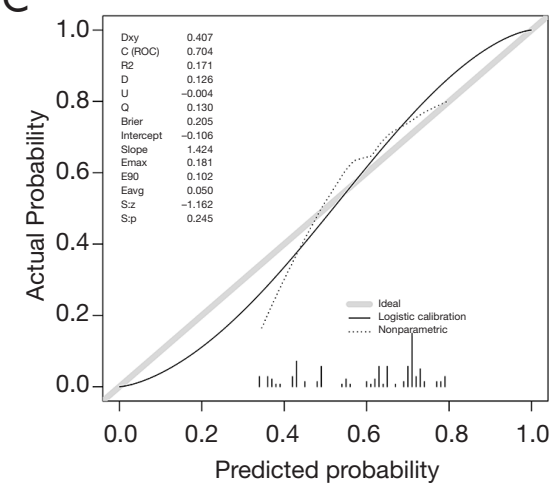

Figure 3 Calibration plots of the nomogram for the probability of bone metastatic patients with breast cancer in the development group and validation groups. (A) SEER development group; (B) SEER validation group; (C) clinical validation group. $\mathrm{P}$ value $=0.982$ vs. 0.662 vs. 0.245 .

which was consistent with the rule of subtypes distribution reported by Yang (24). HR-/HER-2+ and triple-negative breast cancer more often metastasize to visceral organs, it may explain that those patients have died of visceral metastases before bone metastases were noted $(21,25)$.

The nomogram showed that ILC was more likely to develop bone metastasis than infiltrative ductal carcinoma (IDC). In the United States, ILC accounts for $10-15 \%$ of all breast cancer, while in China accounts for $2.1-2.6 \%$ of all breast cancer. Because of deficient research data, the mechanism of tumor metastasis has not been thoroughly studied. Previous study reported that comparing to IDC, ILC was usually diagnosed in an advanced stage, with larger tumor size and more positive lymph nodes $(26,27)$. Some studies showed different prognosis of ILC $(28,29)$. It is reported that $80 \%$ of ILC cells have completely lost the expression of E-cadherin causing reduce intercellular adhesion, and P120 staining showed a deficiency of cell membrane expression (30). Aromatase inhibitors were recommended for postmenopausal ILC patients (31), but the high-risk performance of tumor indicated that we need to consider stronger endocrine therapy and/or other comprehensive treatment for bone metastases of ILC.

Age and histologic grades were independent prognostic factor for the occurrence of bone metastases. Purushotham and James $(32,33)$ have reported age and grade also were significant predictors results in different prediction models. We demonstrated that young/low histologic grades patients were more prone to develop bone metastases. There are few studies on this issue at present, and additional experimental data are needed.

In our study, young age and HR+/HER-2 - subtype were prognostic factors for increasing incidence of bone metastases. According to the results of the TAILORx 
trial, early breast cancer patients with HR+/HER2-, negative axillary lymph nodes, less than 50 years old and a recurrence score of 11-15 could benefit from adjuvant chemotherapy (34). Although it was a trial based on early breast cancer patients, it still inspired us that those advanced $\mathrm{HR}+/ \mathrm{HER} 2$ - young breast cancer patients ( $<50$ years old) with moderate risk of recurrence detected by 21 genes test would benefit from adjuvant chemotherapy to reduce the risk of bone metastasis. In addition, taking the joint experiment of Text/soft into account, young breast cancer patients with $\mathrm{HR}+$ should not only strengthen chemotherapy, but also strengthen adjuvant endocrine therapy to prolong disease free survival. Besides, the systemic treatment regimens for bone metastases will be more precise with the refinement of biological factors. Further researches will be expected.

As shown in Figure 1, surgery could significantly reduce the risk of bone metastasis compared with those without surgery. In addition, it has been known that resection of the tumor with negative surgical margin will reduce the tumor burden and improve prognosis of patients (35). A recent phase III trial (E2108) reported the meaningful results that there was no significant difference for stage IV breast cancer in $\mathrm{OS}(\mathrm{P}=0.63)$ or progression-free survival $(\mathrm{P}=0.40)$ comparing optimal systemic therapy (OST) and locoregional treatment (LRT) with OST alone arm. However, there were much likelihood of locoregional recurrence/progression and the worse health-related quality of life both in the OST (36). This provided us with a new therapeutic idea for decreasing the risk of incidence of breast cancer bone metastasis and relieving the SREs. For young/HR+/HER2- patients who can tolerate surgery and in whom the resectable tumor with negative resection margin, surgery may be not only a treatment to relieve local complications, but also would be a method to reduce tumor burden. As for prolonging the survival of patients with distant metastases, there will need more sufficient evidence to prove. The SEER database lacks patient basic data, lifestyle, genetic testing, and other information, which are important factors causing the difference in survival rates. Furthermore, a nomogram needs to contain more risk variables to increase credibility.

\section{Conclusions}

The nomogram as proposed in this study objectively and accurately predicted the risk of breast cancer patients with bone metastases. In this study, young patients,
$\mathrm{HR}+$ subtype, low histological grade, ILC, and primary breast site without surgery were susceptibility factors for the incidence of skeletal metastases. For these highrisk patients, we should take early identification and effective measures during the follow-up and treatment to control tumor growth and prolong the survival time of patients, and delay progression of SREs at the same time. Additional studies are required to determine whether it can be applied to other patient groups.

\section{Acknowledgments}

Funding: The work was supported by Subject Development Project of China Medical University (No. 3110117053 to $\mathrm{BC})$. The funders had no role in the study design, collection and analysis of the data, decision to publish, or manuscript preparation.

\section{Footnote}

Reporting Checklist: The authors have completed the TRIPOD reporting checklist. Available at http://dx.doi. org/10.21037/tcr-20-2379

Peer Review File: Available at http://dx.doi.org/10.21037/tcr20-2379

Conflicts of Interest: All authors have completed the ICMJE uniform disclosure form (available at http://dx.doi. org/10.21037/tcr-20-2379). The authors have no conflicts of interest to declare.

Ethical Statement: The authors are accountable for all aspects of the work in ensuring that questions related to the accuracy or integrity of any part of the work are appropriately investigated and resolved. The study was conducted in accordance with the Declaration of Helsinki (as revised in 2013). This article does not contain any studies with human participants or animals performed by any of the authors. All procedures performed in study involving human data were extracted freely from the SEER Research Data available to the public online (https://seer.cancer.gov/ data/access.html).

Open Access Statement: This is an Open Access article distributed in accordance with the Creative Commons Attribution-NonCommercial-NoDerivs 4.0 International License (CC BY-NC-ND 4.0), which permits the non- 
commercial replication and distribution of the article with the strict proviso that no changes or edits are made and the original work is properly cited (including links to both the formal publication through the relevant DOI and the license). See: https://creativecommons.org/licenses/by-nc-nd/4.0/.

\section{References}

1. Siegel RL, Miller KD, Jemal A. Cancer statistics, 2019. CA Cancer J Clin 2019;69:7-34.

2. Ibrahim T, Mercatali L, Amadori D. A new emergency in oncology: Bone metastases in breast cancer patients (Review). Oncol Lett 2013;6:306-10.

3. Lipton A, Theriault RL, Hortobagyi GN, et al. Pamidronate prevents skeletal complications and is effective palliative treatment in women with breast carcinoma and osteolytic bone metastases: long term follow-up of two randomized, placebo-controlled trials. Cancer 2000;88:1082-90

4. Briasoulis E, Karavasilis V, Kostadima L, et al. Metastatic breast carcinoma confined to bone: portrait of a clinical entity. Cancer 2004;101:1524-8

5. Solomayer EF, Diel IJ, Meyberg GC, et al. Metastatic breast cancer: clinical course, prognosis and therapy related to the first site of metastasis. Breast Cancer Research Treatment 2000;59:271-8.

6. Wedin R, Bauer HC, Rutqvist LE. Surgical treatment for skeletal breast cancer metastases: a population-based study of 641 patients. Cancer 2001;92:257-62.

7. Harries M, Taylor A, Holmberg L, et al. Incidence of bone metastases and survival after a diagnosis of bone metastases in breast cancer patients. Cancer Epidemiol 2014;38:427-34.

8. Erdogan B, Cicin I. Medical treatment of breast cancer bone metastasis: from bisphosphonates to targeted drugs. Asian Pac J Cancer Prev 2014;15:1503-10.

9. D’Oronzo S, Coleman R, Brown J, et al. Metastatic bone disease: Pathogenesis and therapeutic options: Up-date on bone metastasis management. J Bone Oncol 2019;15:004-4

10. Press DJ, Miller ME, Liederbach E, et al. De novo metastasis in breast cancer: occurrence and overall survival stratified by molecular subtype. Clin Exp Metastasis 2017;34:457-65.

11. Wei B, Wang J, Bourne $\mathrm{P}$, et al. Bone metastasis is strongly associated with estrogen receptor positive/progesterone receptor negative breast carcinomas. Hum Pathol 2008;39:1809-15.

12. Otto MF, Zhuoxin S, Giuseppe V, et al. Patterns of Recurrence and outcome according to breast cancer subtypes in lymph node-negative disease: results from international breast cancer study group trials VIII and IX. J Clin Oncol 2013;31:3083-90.

13. Ding L, Liu G, Zhang X, et al. A deep learning nomogram kit for predicting metastatic lymph nodes in rectal cancer. Cancer Med 2020. [Epub ahead of print].

14. Hu J, Jiao X, Zhu L, et al. Establishment and verification of the nomogram that predicts the 3-year recurrence risk of epithelial ovarian carcinoma. BMC Cancer 2020;20:938-47.

15. Ma W, Wang X, Xu G, et al. Distant metastasis prediction via a multi-feature fusion model in breast cancer. Aging 2020;12:18151-62.

16. Sternberg CN: Are nomograms better than currently available stage groupings for bladder cancer? J Clin Oncol 2006;24:3819-20.

17. Mariani L, Miceli R, Kattan MW, et al. Validation and adaptation of a nomogram for predicting the survival of patients with extremity soft tissue sarcoma using a threegrade system. Cancer 2005;103:402-8.

18. Wang L, Hricak H, Kattan MW, et al. Prediction of organ-confined prostate cancer: Incremental value of MR imaging and MR spectroscopic imaging to staging nomograms. Radiology 2006;238:597-603.

19. Surveillance, Epidemiology, and End Results (SEER) Program Research Data, National Cancer Institute, DCCPS, Surveillance Research Program, released April 2018, based on the November 2017 submission.1973-2015.

20. Abdel-Rahman O, Validation of the 8th AJCC prognostic staging system for breast cancer in a population-based setting. Breast Cancer Res Treat 2018;168:269-75.

21. Hagberg KW, Taylor A, Hernandez RK, et al. Incidence of bone metastases in breast cancer patients in the United Kingdom: results of a multi-database linkage study using the general practice research database. Cancer Epidemiol 2013;37:240-6.

22. Ouldamer L, Bendifallah S, Chas M, et al. Intrinsic and extrinsic flaws of the nomogram predicting bone-only metastasis in women with early breast cancer: An external validation study. Eur J Cancer 2016;69:102-9.

23. Delpech Y, Bashour SI, Lousquy R, et al. Clinical nomogram to predict bone-only metastasis in patients with early breast carcinoma. Br J Cancer 2015;113:1003-9.

24. Yang $H$, Wang R, Zeng F, et al. Impact of molecular subtypes on metastatic behavior and overall survival in patients with metastatic breast cancer: A single-center study combined with a large cohort study based on the Surveillance, Epidemiology and End Results database. 
Oncol Lett 2020;20:87.

25. Schwentner L, Wöckel A, König J, et al. Adherence to treatment guidelines and survival in triple-negative breast cancer: a retrospective multi-center cohort study with 9156 patients. BMC Cancer 2013;13:487.

26. Chen Z, Yang J, Li S, et al. Invasive lobular carcinoma of the breast: A special histological type compared with invasive ductal carcinoma. PLoS One 2017;12:e0182397.

27. Cristofanilli M, Gonzalez-Angulo A, Sneige N, et al. Invasive lobular carcinoma classic type: response to primary chemotherapy and survival outcomes. J Clin Oncol 2005;23:41-8.

28. Azim HA, Malek RA, Azim HA Jr. Pathological features and prognosis of lobular carcinoma in Egyptian breast cancer patients. Womens Health (Lond) 2014;10:511-8.

29. Adachi Y, Ishiguro J, Kotani H, et al. Comparison of clinical outcomes between luminal invasive ductal carcinoma and luminal invasive lobular carcinoma. BMC Cancer 2016;16:248.

30. Ciriello G, Gatza ML, Beck AH, et al. Comprehensive Molecular Portraits of Invasive Lobular Breast Cancer. Cell 2015;163:506-19.

31. Farese SA, Aebi S. Infiltrating lobular carcinoma of the

Cite this article as: Ye LJ, Suo HD, Liang CY, Zhang L, Jin ZN, Yu CZ, Chen B. Nomogram for predicting the risk of bone metastasis in breast cancer: a SEER population-based study. Transl Cancer Res 2020;9(11):6710-6719. doi: 10.21037/tcr20-2379 breast: systemic treatment. Breast Dis 2008-2009;30:45-52.

32. Purushotham A, Shamil E, Cariati M, et al. Age at diagnosis and distant metastasis in breast cancer-a surprising inverse relationship. Eur J Cancer 2014;50:1697-705.

33. James JJ, Evans AJ, Pinder SE, et al. Bone metastases from breast carcinoma: histopathological - radiological correlations and prognostic features. Br J Cancer 2003;89:660-5.

34. Sparano JA, Gray RJ, Makower DF, et al. Clinical Outcomes in Early Breast Cancer With a High 21-Gene Recurrence Score of 26 to 100 Assigned to Adjuvant Chemotherapy Plus Endocrine Therapy: A Secondary Analysis of the TAILORx Randomized Clinical Trial. JAMA Oncol 2020;6:367-74.

35. Singletary SE, Walsh G, Vauthey JN, et al. A role for curative surgery in the treatment of selected patients with metastatic breast cancer. Oncologist 2003;8:241-51.

36. Seema AK, Fengmin Z, Lawrence JS, et al. A randomized phase III trial of systemic therapy plus early local therapy versus systemic therapy alone in women with de novo stage IV breast cancer: A trial of the ECOG-ACRIN Research Group (E2108). J Clin Oncol 2020;38:abstr LBA2. 